\title{
La enseñanza mediante la gamificación
}

\section{Teaching through gamification}

\author{
Enrique Espinoza Freire \\ Universidad Técnica de Machala, Machala, Ecuador \\ eespinoza@utmachala.edu.ec
}

Fecha de recepción: 19/01/2018

Fecha de aceptación: 11/03/2018

Publicado: 26/12/2018

\section{Resumen}

El análisis de las ventajas que puede tener la gamificación de la enseñanza frente a las formas reproductivas y mecanicistas de la metodología tradicional es el objetivo de esta comunicación; basada en un estudio bibliográfico, una construcción de un diagnóstico y la interpretación de esos datos, que permitió arribar a conclusiones sobre la ventaja de los juegos virtuales en tanto contribuyen a la elevación de la eficacia de la docencia, pues activan la motivación, el interés y la reflexión consciente. Los fundamentos analizados indican lo beneficioso que resulta la aplicación de estos recursos tecnológicos y la predicción de la posibilidad de eficiencia y validez de los videojuegos en el aprendizaje de la temática "El Sistema Solar", que ha sido evaluada a partir de los resultados deficientes de la metodología tradicional empleada en la enseñanza de este contenido de la asignatura de Estudios Sociales. Palabras clave: gamificación, videojuegos, aprendizaje, estudios sociales.

\begin{abstract}
The analysis of the advantages that can have the gamification of the teaching in front of the reproductive forms and mechanics of the traditional methodology is the objective of this communication; based on a bibliographical study, a construction of a diagnosis and the interpretation of those data that it allowed to arrive to conclusions on the advantage of the virtual games as long as they contribute to the elevation of the effectiveness of the teaching,
\end{abstract}


because they activate the motivation, the interest and the conscious reflection. The analyzed foundations indicate the beneficial thing that it is the application of these technological resources and the prediction of the possibility of efficiency and validity of the video games in the learning of the thematic one "The Solar System" that has been evaluated starting from the faulty results of the methodology traditional employee in the teaching of this content of the subject of Social Studies.

Key words: gamification, video games, learning, social studies.

\section{Introducción}

La sociedad contemporánea vive grandes cambios producto al desarrollo científico-técnico alcanzado y es indispensable formar parte de los mismos, una de las líneas para lograrlo es la incorporación a la educación del uso de las tecnologías de la información y la comunicación (TIC); esta inclusión está produciendo un cambio de paradigma en la concepción de cómo se debe enseñar y aprender; pasando del aprendizaje centrado en el profesor al aprendizaje centrado en el estudiante (Martín, 2011a); en este sentido los software didácticos ocupan un importante rol y en particular los videojuegos; su presencia en el proceso de enseñanzaaprendizaje puede constituir una estrategia metodológica dentro de la asignatura de Estudios Sociales. Se ha insistido en la centralidad que la enseñanza-aprendizaje debe poner en el alumno, al respecto algunos autores señalan que ese aprendizaje tenga como eje el desarrollo de las competencias, entendidas como el desarrollo de capacidades personales (López, 2011; Martín, 2011b).

Mediante esta metodología innovadora se pueden obtener resultados superiores en la construcción del conocimiento, al convertir al estudiante en protagonista de su propio aprendizaje. Algunos autores apuestan a ella dentro de la asignatura de Estudios Sociales al visualizar una nueva perspectiva basada en la motivación e interés por la asignatura, 
vinculada con el encanto de los juegos (Guerra, 2013). Sin embargo, esta visión limitada a los aspectos motivacionales coloca al proceso de enseñanza-aprendizaje en una situación problémica al privilegiar solo una parte de los elementos del proceso educativo y obviar los objetivos, los contenidos, la evaluación; conviene revisar la fundamentación al respecto y ordenar esa nueva visión de la enseñanza.

Para alcanzar este propósito lo primero es localizar quienes y qué posturas se asumen al respecto; interesa así conocer donde se fundamentan los videojuegos como entornos de aprendizaje (Contreras, Eguia \& Solano, 2011), a lo cual se une una teoría de la educación basada en competencias que enfatiza el desarrollo constructivo de habilidades, conocimientos y actitudes. Para otros los videojuegos son entornos inmersivos que permiten experiencias personales, epistemológicas, culturales y conexiones potencialmente transformadoras (Williamson, Squire, Halverson \& Gee, 2005); las aplicaciones de esas experiencias contribuirán a un cambio positivo en el aprendizaje de la asignatura de Estudios Sociales, mediante el uso de los videojuegos.

\section{Desarrollo}

El uso de videojuegos alcanza un valor pedagógico al reemplazar materiales de estudio lo que permite la trasmisión de los conocimientos de manera más ágil y divertida (Contreras \& Eguia, 2016). Los juegos ocupan un lugar central en la vida de los infantes, es por ello, que el buen empleo de software educativos permiten combinar lo lúdico con lo cognoscitivo al potenciar el desarrollo de habilidades sensoriales, perceptuales, cognitivas e informáticas, que tanto necesitan los niños(as) en sus relaciones sociales (Oliva, 2011; Montell, Leyva \& Castillo, 2013). Estos medios tecnológicos propician que los alumnos capten mayor información, sean más ágiles en interpretarla y la almacenen a largo plazo gracias a la atención que despierta la lucha por el posicionamiento exitoso, que les obliga a prestar atención y concentración en cada una de las acciones que realizan para llegar al objetivo del 
juego, incrementar el nivel de reflexión y crítica (García, Barrio, Medina, Arroyo \& Gálvez, 2011).

Para la introducción de los softwares en el ámbito educativo es necesaria la preparación de los docentes; según Martínez (2007), el estudiante domina las TIC, sin embargo, los docentes no se encuentran preparados para su empleo. Esta realidad impone un reto ineludible al docente, la preparación metodológica necesaria para su óptimo desempeño en la formación de las nuevas generaciones donde las TIC se convierten en una exigencia permanente como resultado de su propio desarrollo (Rivera, 2011). Sin embargo, es pertinente señalar que el docente no debe dejarse deslumbrar por estas tecnologías, debe pensar siempre en adaptarlas a la enseñanza, no la enseñanza a éstas (Beteta, 2012); en tal sentido González (2011) señala que el maestro ha de ser más experto en enseñar que en las TIC, lo que no impide, de ser posible, el dominio de ambas áreas. Debe ser crítico con las nuevas tecnologías y recordar que no son malas ni buenas en sí mismas, todo depende de cómo y para qué se usan.

El juego virtual exige una adaptación a los contextos, donde requiere ser lo más humanamente flexible para asegurar el aprendizaje, esa flexibilidad se sustenta en generar motivación en la adquisición de conocimientos mediante los videojuegos como entornos de aprendizaje y su aplicación en el mundo educativo, posibilidad que puede propiciar la gamificación en tanto es una nueva tendencia educativa que incita a los estudiantes a mantener un compromiso con las diversas asignaturas establecidas en su pensum de estudio, ya que encuentran interés en la diversidad de actividades que se pueden realizar por medio de aplicaciones y sitios (Contreras, Eguia \& Solano, 2011; Manrique, 2013), principalmente orientados a construir conocimientos significativos en los educandos, lo que transforma el aprendizaje monótono en activo (Parente, 2016), crea un ambiente genuinamente innovador y tecnológico, adapta los contenidos a las exigencias de la educación contemporánea: 
desarrollar una forma de trabajo cooperativo y colaborativo. En sentido general el software educativo potencia en los estudiantes el trabajo autónomo, la capacidad de búsqueda de información y las habilidades de investigación. Se estimula la reflexión y la discusión entre los estudiantes (Fernández \& Torres, 2015). Según Romero (2011), el aprendizaje autónomo es más significativo y activo porque convierte a los estudiantes en personas críticas, constructivas y creativas.

Los juegos virtuales resultan un factor innovador para la resolución de problemas en contextos reales al permitir ajustar las respuestas en el entorno virtual a eventos concretos (Rodríguez \& Santiago, 2015), hecho que los convierte en una tecnología de participación de los alumnos. Comezaña \& García, (2005) consideran que su presencia en la enseñanza ha dado lugar a las aulas virtuales, definidas como un espacio de aprendizaje en línea donde aprendices y tutores interactúan soportados por medios digitales o electrónicos.

Estos fundamentos determinaron la realización de la investigación educativa dirigida a comprobar la tesis de que los videojuegos son entornos que permiten a los alumnos realizar experiencias personales, epistemológicas, culturales y conexiones potencialmente transformadoras (Williamson, Squire, Halverson \& Gee, 2005). Razones que llevaron a concebir el uso de videojuegos en la asignatura de Estudios Sociales, para propiciar cambios positivos en la formación y aprendizaje de los estudiantes.

En base al análisis de estos presupuestos teóricos se orientó la investigación a delimitación del nivel de conocimiento de los estudiantes en la temática "El Sistema Solar" en diversas instituciones educativas de quinto y sexto años de Educación General Básica, mediante la aplicación de un cuestionario a una muestra de 112 estudiantes, seleccionados aleatoriamente mediante el muestreo estratificado, para sobre los resultados obtenidos proponer el uso de la gamificación como estrategia metodológica en el desarrollo de esta temática de la asignatura de Estudios Sociales, visión que tiene en cuenta como esta 
tecnología permite nuevos métodos que motivan el aprendizaje, posibilitan crear ambientes educativos más interactivos y dinámicos. En tal sentido se planteó como problema de investigación: ¿Qué herramienta virtual es posible aplicar en la asignatura de Estudios Sociales en quinto y sexto años de Educación Básica para optimizar el aprendizaje de los estudiantes sobre "El Sistema Solar”?

Se plantea como objetivo: "Optimizar el aprendizaje de la temática "El Sistema Solar" en la asignatura de Estudios Sociales mediante la aplicación de herramientas virtuales que generen un aprendizaje significativo en los estudiantes de quinto y sexto años de Educación General Básica.”

La hipótesis que sustenta esta investigación está concebida a partir de la comprensión de que en razón de la tecno-educación como contribución al fomento del trabajo autónomo y el aprendizaje activo; se propone que los videojuegos, como estrategia metodológica virtual, son propicios para el estudio de la variedad de temáticas que contiene el currículo del tema "El Sistema Solar".

Los antecedentes de este estudio se enmarcan en el proyecto pedagógico "Gamestar (t)" de la asociación ARSGAMES surgido en 2010 con la finalidad de crear un nexo entre la enseñanza, las artes y los videojuegos; el mismo impulsó la utilización de una nueva herramienta pedagógica, que permitiera hacer uso de las nuevas tecnologías al mismo tiempo que se desarrolla la creatividad, criticidad, la autonomía del pensamiento, los valores e intereses de los estudiantes (Cabañes \& Rubio, 2013).

También constituye un antecedente el proyecto "Class of Clans" (Juego de estrategia online) para incrementar la motivación del alumnado hacia el aprendizaje (Echegaray, Espinosa y Ladrón, 2016), que involucró las asignaturas: ciencias naturales, ciencias sociales, tecnología, educación plástica y visual donde los estudiantes lograron aprendizajes de forma integral. Con la aplicación de este proyecto se logró mejorar notablemente el rendimiento 
académico de los estudiantes en las distintas asignaturas integradas, además se incrementó el nivel de motivación, la atención a la diversidad, el trabajo cooperativo y la inteligencia emocional.

Otro de los antecedentes es la investigación realizada por Montell, Leyva \& Castillo, (2013) donde propone el software educativo "Ratín" para favorecer al vocabulario activo y pasivo, a partir de la incorporación de nuevas palabras y potenciar el desarrollo de habilidades informáticas.

La Investigación de campo fue desarrollada en los centros educativos del cantón Machala: "Héctor Encalada Sánchez", "Unión Nacional de Educadores” y "Jesucristo es el Rey" , así como en la institución educacional "Abdón Calderón” del cantón Pasaje, que tributaron la información obtenida mediante la aplicación de un cuestionario elaborado para identificar la situación de aprendizaje, que presentaba el contenido elegido y evaluar las posibilidades de uso de la estrategia metodológica basada en la gamificación para el estudio de la temática "El Sistema Solar".

\section{Metodología}

La orientación de la investigación está dirigida a delimitar el nivel de conocimiento de los estudiantes de quinto y sexto años de Educación General, en la temática "El Sistema Solar", propósito que se aspira lograr mediante la aplicación de un cuestionario a una muestra de 112 estudiantes, seleccionados aleatoriamente mediante el método estratificado, de distintas instituciones escolares, considerando cada una como un estrato, y en consecuencia proponer el uso de la gamificación como estrategia metodológica para la asignatura de Estudios Sociales. Se empleó la revisión bibliográfica para construir el marco referencial y los antecedentes del estudio; así como recursos de la estadística descriptiva: frecuencias absolutas 
y relativas, la media aritmética, tablas y gráficos, que facilitaron el procesamiento y análisis de los datos.

\section{Análisis e interpretación de resultados}

Para obtener un mapa del nivel de información que poseen los estudiantes se resumieron los resultados obtenidos en las calificaciones de cada una de las respuestas de las preguntas del cuestionario aplicado a los 112 estudiantes, en los mismos se le asignó un punto a cada ítem y fue valorado sobre un puntaje total de 20 puntos.

Tabla 1.

Resultados del cuestionario aplicado en la encuesta

$\begin{array}{lll}\mathbf{N}^{\circ} & \text { Preguntas } & \text { R. C. } \%\end{array}$

Íte

m

$1 \quad$ El sistema planetario al que pertenece nuestro planeta se denomina $88 \quad 72,1$ Sistema Solar porque el Sol es la estrella central.

$2 \quad$ El Sistema Solar está conformado únicamente por planetas y estrellas. $\quad 27 \quad 22,1$ 
$7 \quad$ Venus es el único planeta que gira en un movimiento contrario al resto $\quad 49 \quad 40,1$ de planetas.

8 Neptuno también es llamado "planeta rojo".

$60 \quad 49,1$

9 Saturno es el único planeta con anillos visibles desde la Tierra.

$84 \quad 68,8$

10 Mercurio, Venus, Tierra y Marte son planetas rocosos.

$64 \quad 52,4$

6

11 La luna es el único satélite del Sistema Solar $48 \quad 39,3$

12 Las estrellas tienen luz propia.

13 Todos los planetas giran alrededor del sol a la misma velocidad que la $43 \quad 35,2$ Tierra.

14 ¿Un eclipse de sol ocurre cuando la Luna se sitúa entre la Tierra y el $62 \quad 50,8$ Sol?

15 ¿Un eclipse lunar ocurre cuando Venus se ubica entre la Tierra y la $50 \quad 40,9$ Luna?

16 Nuestro Sistema Solar está ubicado en una galaxia llamada Vía Láctea. $\quad \begin{array}{ll}71 & 58,2\end{array}$ 
17 El Sistema Solar tiene 10 planetas principales.

6150,0

18 Júpiter es el planeta más grande del Sistema Solar.

Fuente: Encuesta centros educativos

Según la información ofrecida por la tabla 1, la mayor cantidad de respuestas correctas correspondieron a los ítems 1 y 20; 88 (72,13\%) estudiantes reconocieron el sistema planetario al que pertenece la Tierra y que este es el único planeta con vida; $87(71,31 \%)$ de los examinados clasificaron a Plutón como un planeta enano y conocen que las estrellas tiene luz propia; $84(68,85 \%)$ de los alumnos afirmaron que Saturno es el único planeta con anillos visibles desde la Tierra; estos son los resultados más significativos de respuestas correctas; el resto de las respuestas acertadas estuvo entre un $62,30 \%$ (76) y $22,13 \%$ (27); lo que demuestra en el orden descriptivo que existen insuficiencias en el conocimiento de las características del Sistema Solar.

Tabla 2.

Calificaciones de los estudiantes

\begin{tabular}{lllllllllllllll}
\hline CALIFICACIÓN & 5 & 6 & 7 & 8 & 9 & 10 & 11 & 12 & 13 & 14 & 15 & 16 & 17 & TOTAL
\end{tabular}

$\begin{array}{lllllllllllllll}\text { CANTIDAD DE } & 1 & 2 & 1 & 2 & 6 & 13 & 22 & 18 & 17 & 16 & 9 & 3 & 2 & 112\end{array}$

\section{ESTUDIANTES}

Fuente: Encuesta centros educativos 


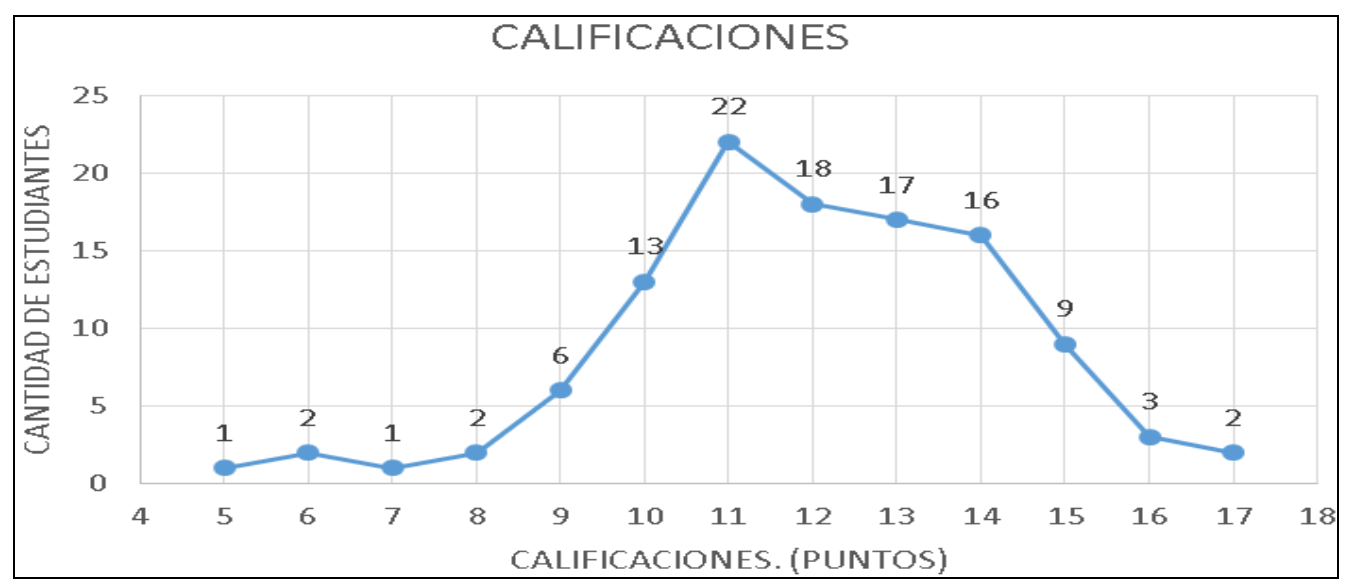

Figura 1. Calificaciones obtenidas por los estudiantes

De acuerdo a la tabla 2 y gráfico 1 , se observa que la calificación más alta obtenida es de 17 sobre 20 puntos lograda por dos estudiantes, lo que significa que dominaron el $85 \%$ de los conocimientos evaluados, mientras que la calificación más baja es de 5 puntos conseguida por un alumno, lo que representa el $25 \%$ de los ítems. Por otro lado, el promedio de las calificaciones es de 12 puntos, alcanzado o superado por $65(58,03 \%)$ estudiantes. Solo resultaron aprobados $30(26,78)$ de los estudiantes. Información que devela insuficiencias en el conocimiento y dominio de los contenidos de la temática "El Sistema Solar", lo cual está causado por factores asociados al interés y comprensibilidad de los conocimientos, así como a un aprendizaje memorístico que no fija suficientemente los conceptos enseñados.

\section{Conclusiones}

Las reflexiones generadas en esta ponencia explican las ventajas que puede tener la gamificación frente a las formas reproductivas y mecanicistas con que la metodología tradicional despliega el proceso de enseñanza-aprendizaje. Los juegos virtuales no son toda la solución del aprendizaje, pero constituyen una contribución a la elevación de la eficacia de la docencia, pues activan la motivación, el interés y la reflexión consciente al estar vinculados mediante el rol de participante en un juego. 
La nueva generación ha sido formada en una ecología tecnológica por lo cual ha creado hábitos relacionados con la manipulación de software y herramientas digitales, factores que deben incrementar el entusiasmo y deseos hacia todo lo relacionado con la tecnología y entornos digitales, en los cuales los videojuegos bien utilizados son una eficaz herramienta pedagógica.

La predicción de la posibilidad de eficiencia y validez de los videojuegos en el aprendizaje de la temática "El Sistema Solar" ha sido evaluada a partir de los resultados insuficientes de la metodología tradicional de enseñar este contenido lo cual induce la necesidad de aplicarlo al nivel de una experiencia con vista a comprobar los supuestos aportados en este estudio.

\section{Recomendaciones}

Estas razones, los presupuestos teóricos ya analizados y los estudios antecedentes realizados sobre la aplicación de software didáctico en la enseñanza, conducen a recomendar:

Una metodología basada en videojuegos para incrementar las vías de trasmisión de conocimientos al utilizar además de los textos los medios audiovisuales para redimensionar la percepción de los contenidos del tema "El Sistema Solar" y de esa manera obtener un mayor rendimiento y dominio de los conocimientos.

Desarrollar un experimento pedagógico para validar en la práctica la metodología basada en el empleo de un videojuego en el tratamiento de tema "El Sistema Solar".

\section{Referencias bibliográficas}

Beteta, S. M. (2012). ¿Profesor TIC o profesor TAC; Lima, Perú: Universidad peruana de Ciencias Aplicadas. Recuperado de https://docs.google.com/a/nyit.edu/file. 
Cabañes, E. \& Rubio, M. (2013). GAMESTAR (T)- An ARSGAMES Project. Recuperado de http://euridicecabanes.es.tl/Gamestar-k1-t-k2--_-An-ARSGAMES-project.htm

Comezaña, O. \& García, F. (2005). Plataformas para educación basada en web: Herramientas, procesos de evaluación y seguridad. Salamanca: Universidad de Salamanca. Recuperado de http://tejo.usal.es/inftec/2005/DPTOIA-ITtejo.usal.es/inftec/2005/DPTOIA-IT-2005-001.pdf.

Contreras, E. R. \& Eguia, J. L. (2016). Gamificación en aulas universitarias. Barcelona, España: Universitat Autonoma de Barcelona. Institut de la Comunicació (InCom). Recuperado de http://incom.uab.cat/download/eBook_incomuab_gamificacion.pdf

Contreras, E. R., Eguia, G. J. \& Solano A. L. (2011). Videojuegos como un entorno de aprendizaje. Revista de Comunicación y Tecnologías emergentes, ICONO14, 9(2), 249-261. Doi: 10.7195/ri14.

Echegaray, J. P., Espinosa, J. \& Ladrón, C. (2016). Class of Clans. Gamificando cuatro asignaturas en un viaje emocionante. Telefónica Explorador de Innovación educativa. Recuperado de http://innovacioneducativa.fundaciontelefonica.com/blog/2016/02/22/class-of-clansgamificando-cuatro-asignaturas-en-un-viaje-emocionante/

Fernández, B. M. \& Torres, G. J. (2015). Actitudes docentes y buenas prácticas con TIC del profesorado de Educación Permanente de Adultos en Andalucía. Universidad Complutense Revista Complutense de Educación, 26, Especial.

García, G. F., Barrio, G. F., Medina, D., Arroyo, G. R. \& Gálvez, C. M. (2011). Señas de identidad del "nativo digital". Una aproximación teórica. Revista Científica Complutenses Cuadernos de Documentación Multimedia, 22.

González, C. (2011). “Tic Tac. Formas de enseñar vs. Maneras de aprender”. Página Web: Los docentes las TIC. Recuperado de 
http://docentesytic.wordpress.com/2011/05/03/tic-tac-formas-de-ensear-vs-manerasde-aprender/

Guerra, M. (2013). La Tecnología y la Educación personalizada: Teoria y Práctica. Mexico DF, México: Ajfaomega.

López, R., J. (2011). Un giro copernicano en la enseñanza universitaria: formación por competencias. Revista de Educación, 356, sept.-dic, 279-301.

Manrique, V. (2013). Gamification y educación: la revolución de aprender jugando. Recuperado de http://comunidad.iebschool.com/iebs/gamification-narrativatransmedia/gamification-y-educacion/

Martín-Cuadrado, A. (2011a). Competencias del estudiante autorregulado y los estilos de aprendizaje. Revista Estilos de Aprendizaje, 8(8).

Martín-Cuadrado, A. (2011b) Desarrollo de las competencias informáticas y la ciudadanía del siglo XXI, (cap. 4). En D.Barros; C. Neves; F. Barreto; J.A. Marques; S. Henriques (Coords.) Educação e tecnologías: reflexão, inovação e práticas. Lisboa, Portugal. Recuperado de http://livroeducacaoetecnologias.blogspot.com/.

Martínez, A., J. (2007). ¿Quién quiere ser profesor? II parte. El Observatodo. Recuperado de http://www.elobservatodo.cl/admin/render/noticia/8972.

Montell, R. I., Leyva P., D. \& Castillo, R.(2013). Sofware educativo "RATÍN”, Camagüey, Cuba: Departamento de Desarrollo de Recursos Informáticos para el Aprendizaje, adscrito a la Universidad de Ciencias Pedagógicas José Martí. Revista electrónica EcuRed. Recuperado de https://www.ecured.cu/Software:Rat\%C3\%ADn

Oliva R., M. (2011). Jugar "sin pantallas" es lo mejor para el cerebro de los niños pequeños. Medlineplus. Recuperado de http://boletinaldia.sld.cu/aldia/2011/10/21/jugar-sinpantallas-es-lo-mejor-para-el-cerebro-de-los-ninos-pequenos 
Parente, D. (2016). Gamificación en la educación. Gamificación en aulas universitarias. Barcelona: España: Universitat Autónoma de Barcelona. Instituto de la comunicación. Recuperado de http://incom.uab.cat/download/eBook_incomuab_gamificacion.pdf.

Rivera, S. A. (2011). Formación del docente para el uso de las TIC. (Ponencia presentada en el Congreso Internacional EDUTEC 2011, Pechuga Hidalgo, México.

Rodríguez, F. \& Santiago, R. (2015). Gamificación: Cómo motivar a tu alumnado y mejorar el clima en el aula. México D.F., Mexico:Amazon.

Romero, G. R. (2011). Tecnología Educativa Prospectiva. Quito.Ecuador: CODEU:

Williamson, S., D., Squire, K., Halverson, R. \& Gee, J. (2005). Video Games and The future of Learning. Wisconsin:EE,UU: University of Wisconsin-Madison and Academic Advanced Distributed Learning Co-Laboratory. 